Duke primary care clinics and prescribed antihyperglycemic medication. We will recruit through email and phone calls. Enrolled patients will complete the Interpersonal Processes of Care Short Form and Extent of Medication Adherence survey to measure patient perceptions of care (predictor) and medication adherence (secondary outcome). No show appointments and cancellations (secondary outcomes) and most recent hemoglobin Alc (primary outcome) will be collected from the Electronic Medical Record. We will also collect basic demographic information, insurance status, financial security, significant co-morbidities, and number and type (subcutaneous vs oral) of antihyperglycemic medications. RESULTS/ANTICIPATED RESULTS: -The study is powered to detect a $0.6 \%$ difference in $\mathrm{HbA} 1 \mathrm{c}$, our primary outcome, between high and low scorers on the Interpersonal Processes of Care subdomains. -We expect that higher patient scores in the positive domains of the IPC survey and lower DISCUSSION/SIGNIFICANCE OF IMPACT: This study will provide information to develop and implement targeted interventions to reduce racial and ethnic disparities in patients with Type II diabetes. We hope to gain information on potentially modifiable factors in patient-provider interactions that can be intervened upon to improve prevention and long-term outcomes in these populations.

4481

\section{Better Together Harrisburg: Community-Driven Research Day}

Andrea Murray ${ }^{1}$, Dr. Martha Wadsworth, Dr. Jennifer Kraschnewski, Kathleen Best, and Carmen Henry-Harris

${ }^{1}$ Penn State Clinical and Translational Science Institute

OBJECTIVES/GOALS: The overall goal of the Community-Engaged Research Core, supported by the Penn State Clinical and Translational Science Institute, is to invest in opportunities that promote collaboration between researchers and communities. Research in which community members are participating in the research process will more likely lead to reducing health disparities when compared to more traditional approaches. This abstract describes a community research day that brought researchers and community-based organizational leaders together to discuss critical areas of research. We aim to highlight a successful approach for how to work with a community, particularly one that has been distrustful of research, to facilitate and support collaborations between academic researchers and community-based organizational leaders (CBOs). Community-based organizational leaders are often the most knowledgeable individuals when it comes to identifying and discerning the needs and research priorities of their communities and they are generally in the best positions to help build greater trust between academic researchers and communities. METHODS/STUDY POPULATION: A Community Research Day Steering Committee was formed in the spring of 2018 and consisted of 10 communitybased organizational leaders from Harrisburg, Pennsylvania, two Penn State University staff, and one Penn State University faculty member. The Steering Committee's purpose was to design, plan, and execute an event (Better Together: Community Driven Awareness) in which community-organizational leaders and faculty researchers came together to discuss possible research collaborations to improve community health. The Steering Committee participated in bi-monthly planning meetings leading up to the event, Better Together: Community-Driven Awareness. During these planning meetings, members determined that mental health and nutrition were two critical areas deserving of more attention from research within their geographical community. Organizations were asked to identify sub-categories within mental health and nutrition that they saw as most relevant to their communities. The sub-categories that they selected became the theme topics for round table discussions at the main event. This information was also used to determine which academic researchers to invite to the event, based on scientific expertise. In addition to selecting these topics for table discussions, the Steering Committee provided advice on the agenda and program materials. The agenda for Better Together: Community-Driven Awareness featured a presentation from a successful collaboration between a faculty member and a community-based organization whose project was centered around suicide prevention in the school system. After the presentation, researchers and CBOs sat at round tables for facilitated discussions about their table's theme. The facilitated discussions fostered new relationships and led to collaborations outside of the event. Following the round-table discussions, there was a presentation about funding and next steps. Lastly, feedback forms were given to each attendee to assess their experience of the event and to better understand what to improve upon for the future. RESULTS/ ANTICIPATED RESULTS: Following the Community-Driven Awareness event, the Community-Engaged Research Core at Penn State released a call for proposals for planning grants to be awarded to faculty/community-based organization teams. These grants were intended to build capacity for externally-funded research that seeks to address important community-identified research questions. The internal grants support meetings to discuss mutual interests, develop research questions, identify leaders, conduct literature reviews, and collect pilot data. A team must have included, at a minimum, one Penn State faculty researcher and one community-based organizational leader as co-principal investigators. In the proposal, the team was asked to describe its preliminary research question, the work to be accomplished during the planning period, anticipated outcome(s) and deliverables, and preliminary ideas for seeking future external funding. A two-page narrative briefly described how the team members' expertise/experience/constituencies would address the specified research question. In addition, the team provided a budget and budget justification. Planning grants ranged from $\$ 500-\$ 5,000$. Funds were allocated for a 6-12 month period. After the call was sent out, seven proposals were submitted and three were selected for external funding. Proposal topics included: * Exploring the Mechanism of Engagement in HIV Testing, Prevention, and Care Among African American and Hispanic/Latino Men who Have Sex with Men * Educator Translation of a Universal SocialEmotional Learning Program in School Practice * Growing Nutritious Communities: Gardening to increase access to and knowledge about fresh fruits and vegetables among residents in South Harrisburg, Hall Manor community. DISCUSSION/ SIGNIFICANCE OF IMPACT: There are several academic institutions that have implemented similar events whose goal is to bring together academic researchers and community-based organizational leaders. To our knowledge, this is one of a few examples of an event that was developed from the ground up by a committee comprised mostly of community organization leaders. The community leaders guided the decisions made in all phases of the event design from determining the research themes to providing input on program materials. Additionally, our Steering Committee garnered the interest and attendance from over 20 community participating organizations, which attests to their commitment and dedication to seeing 
this event through from beginning to end. The feedback received from the event was overwhelmingly positive. Both academic researchers and community-based organizational leaders expressed their appreciation for an event that brought both parties together in a space where they felt comfortable to share ideas and knowledge. When asked how we could improve this event in the future, most attendees shared that they wanted more time and more opportunities to connect. One limitation of the event noted by attendees was that attendees were not able to sign up for the round table discussions themselves but were placed strategically at them by our Steering Committee. Therefore, at our next event, attendees will be able to select their tables and determine which themed topic they prefer to participate in. Lastly, we are considering how to best summarize the ideas that are generated from these round table discussions in a way that can be shared with the larger group and in a way that might foster collaborations outside of the event.

4258

Black women's narratives: A mixed-methods exploration of microaggressions and mental health

Alexandria Colburn ${ }^{1}$, and Ed de St. Aubin ${ }^{1}$

${ }^{1}$ Marquette University

OBJECTIVES/GOALS: This social justice-oriented, multi-method study aims to gain an understanding of the unique sources of stress and resilience impacting Black women in Milwaukee. As clinical researchers, it is imperative that we understand the mechanisms underlying the relationship between marginalized identities and substantial health disparities. METHODS/STUDY POPULATION: Participants were Black women, diverse in age, income, and sexual orientation to emphasize an intersectional approach (current $\mathrm{N}=87$ of 160). Our interdisciplinary team collected two interrelated data types: narrative and survey. Participants completed a 1.5-hour life story interview in which they were asked to share stories from their lives, their backgrounds, plans for the future, forces that shaped their stories, and how their identities have influenced their experiences. Interviews were done one-on-one and conducted by Black women interviewers. They were also asked to complete an online survey protocol including measures of stress, trauma, microaggressions, coping, and well-being. Transcribed life story interviews will be coded utilizing grounded theory, an intensive qualitative analysis method. RESULTS/ANTICIPATED RESULTS: The presentation will focus on the unique methodological approach, which emphasizes community empowerment through collaboration and cultural competency. Data collection is still in progress, but initial relationships between key variables will be discussed. It is anticipated that greater frequency and appraisal of microaggressions will significantly predict higher reported stress, anxiety, and depression. Within-group diversity will be examined as well. Relevant themes emerging from grounded theory will also be presented. Results will directly inform community outreach aimed at improving the lives of Black women. DISCUSSION/SIGNIFICANCE OF IMPACT: This study sheds light on unique, identity-related stressors believed to contribute to mental and physical health disparities. It also builds on current theories, filling voids in the research literature by taking a psychosocial perspective, emphasizing the voices of participants, and channeling the findings to specific programs in the community.
4449

Building Capacity in the Flint Community in the Midst of the Ongoing Water Crisis

Athena S. McKay ${ }^{1}$, Adam Paberzs ${ }^{2}$, Patricia Piechowski², Donald

Vereen $^{1}$, and Susan Woolford ${ }^{1}$

${ }^{1}$ University of Michigan; ${ }^{2} \mathrm{MICHR}$ Community Engagement

OBJECTIVES/GOALS: Examining the impact of the Building Capacity for Research and Action (BCRA) Award created by the Community Engagement (CE) Program at the Michigan Institute for Clinical \& Health Research (MICHR)-a Clinical \& Translational Science Award (CTSA) site at the University of Michigan-in partnership with Community Based Organization Partners (CBOP). METHODS/STUDY POPULATION: The BCRA is a funding mechanism that supports new communityengaged research (CEnR) partnerships and projects that address community-identified health needs in Flint, Michigan. BCRA projects are required to be Flint-based and inclusive of both community and academic partners. A study section consisting of 10 MICHRaffiliated faculty and community partners reviewed proposals and made funding decisions. Funded teams were trained on Institutional Review Board (IRB) and reporting requirements by CE staff. MICHR provides support to BCRA-funded teams through monthly email correspondence with the CE Flint connector, budget review, mediation, regulatory assurance of IRB and the National Center for Advancing Translational Science (NCATS) requirements, coordinating six-month and final reporting, and hosting an annual stakeholder meet and greet. RESULTS/ANTICIPATED RESULTS: In 2017, the BCRA Award submitted its first request for proposals. It received 20 applications in 2018, and selected eight awardees, providing them with a total of $\$ 60,000$ in funding. Four received $\$ 5,000$ for partnership development and another four received $\$ 10,000$ for their research projects. The BCRA Award received 16 applications in 2019, expanding its academic pool to include the University of Chicago, U-M Flint, Michigan State University, and Michigan State University-Flint in addition to the University of Michigan. Five recipients were selected and received a total of $\$ 45,000$ in funding. One was awarded $\$ 5,000$ for partnership development and another four were awarded $\$ 10,000$ for their research projects. MICHR has invested over $\$ 100,000$ in Flint through this mechanism, which was renewed in 2019. DISCUSSION/SIGNIFICANCE OF IMPACT: Each awardee presented at the annual stakeholder meet and greet. They showcased their projects with a brief overview and spoke about their expectations, lessons learned, partnership strengths and challenges, translational issues, and proposed next steps for subsequent grants, publications.

4405

Chronic Disease in Indiana - Using a Community Health Matrix to Determine Health Factors for Indiana Counties Sarah Wiehe ${ }^{1}$, Aaron Zych ${ }^{2}$, Karen Hinshaw, Ann Alley ${ }^{3}$, Gina Claxton $^{4}$, and Dennis Savaiano ${ }^{5}$

${ }^{1}$ Indiana University School of Medicine; ${ }^{2}$ Indiana University; ${ }^{3}$ Indiana State Department of Health; ${ }^{4}$ Community Health Partnerships; ${ }^{5}$ Purdue University

OBJECTIVES/GOALS: The goal of this project was to inform four chronic disease initiatives, working together on the team Connections IN Health, and counties in Indiana on certain areas of need to assist them in collaborative planning. The chronic diseases 